\title{
Suppression of grating lobes for MMW sparse array setups
}

\author{
S. Bertl, A. Kirschner, and J. Detlefsen
}

Fachgebiet Hochfrequente Felder und Schaltungen, Technische Universitt München, Arcisstr. 21, 80333 München, Germany

\begin{abstract}
For arrays the placement of the single elements determines the angular resolution and the unambiguity interval. The width of the total array determines the resolution capabilities. The wider the elements are placed from each other, the more space in Fourier domain is covered by the measurement and the resolution in time domain will improve. On the other hand the density of the elements has an effect on the angular interval in which objects can be detected unambiguously. For objects within the unambiguous interval grating lobes will appear outside this area while objects outside result in grating lobes in the interval of interest. In this paper the properties of arrays regarding resolution and unambiguity interval will be discussed and methods for the suppression of ambiguous grating lobes are suggested. One approach to suppress the influence of the grating lobes lies in the evaluation of different frequency bands.
\end{abstract}

\section{Introduction}

To realise a sensor with range and azimuth resolution capabilities, a single antenna with high gain that is moved mechanically can be used. To avoid mechanical movements arrays can be considered instead. Their performance depends on the number of elements and their placement. However the hardware effort is considerable higher such that the amount of elements is usually limited. A concept that reduces the effort to a certain part exploits the concept of virtual apertures. This means every combination of transmitter and receiver forms a virtual element of an array that contains overall more elements than the single arrays for transmit and receive. The properties of these arrays and possible applications in MMWdomain are discussed.

Correspondence to: $\mathrm{S}$. Bertl

(sebastian.bertl@tum.de)

\section{Properties of arrays}

\subsection{Concept of virtual arrays}

Virtual arrays can be formed from an arbitrary transmitting array with $N$ antenna elements and a receiving array with $M$ elements. The position of the single antenna elements is known and denoted with $\boldsymbol{x}_{T n}$ for the $n$-th transmitting antenna and $\boldsymbol{x}_{R m}$ for the $m$-th receiving antenna.

For being able to separate the transmitted signals of the different Tx-antennas at the receivers it is required that the transmitted waveforms $s_{i}$ are orthogonal such that

$$
\int s_{n}(t) \cdot s_{m}^{*}(t) d t=\left\{\begin{array}{ll}
1 & n=m \\
0 & n \neq m
\end{array} .\right.
$$

In the receiving antennas the orthogonal waveforms are extracted by $N$ matched filters. The total number of extracted signals is then $N \cdot M$. The received signal of a point scatterer with a reflection coefficient $\rho$ can be written as

$$
\begin{aligned}
s_{m, n}(t) & =\rho \cdot \exp \left(j k_{0} \boldsymbol{u} \cdot\left(\boldsymbol{x}_{T n}+\boldsymbol{x}_{R m}\right)\right) \\
& =\rho \cdot \exp \left(j 2 k_{0} \boldsymbol{u} \cdot\left(\frac{\boldsymbol{x}_{T n}+\boldsymbol{x}_{R m}}{2}\right)\right) .
\end{aligned}
$$

The vector $\boldsymbol{u}$ is the unit vector pointing from the center of the array towards the scattering centre. In this description it is assumed that the distance between the point scatterer and the two arrays is much larger than the dimensions of the array. Equation (3) shows that the response is the same as for an array with $M \cdot N$ elements where each element is transmitting and receiving just its own signal located in the middle of a pair of transmit and receive antenna at

$$
\frac{\boldsymbol{x}_{T n}+\boldsymbol{x}_{R m}}{2} \quad \text { with } m=1 \ldots, M, n=1, \ldots, N .
$$




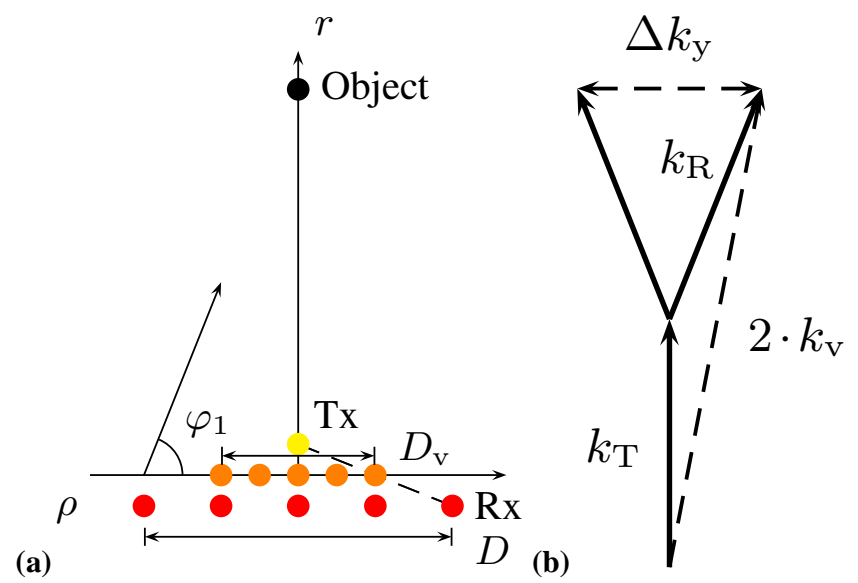

Fig. 1. Virtual array formed of one Tx and $n \mathrm{Rx}$ antennas. (a) Array geometry. (b) k-space representation.

\subsection{Realisation of orthogonal signals}

The separation of the transmitted signals at the receivers is essential for the realisation of virtual arrays. Different approaches can be considered for the orthogonalisation. In general the principles known from communication can be applied for the radar case too. The approach that does not require a high hardware effort is to separate the signals in time by transmitting the signals at a given sequence. The separation of the signals at the receiver is then done by applying rectangular windows to the specific time slots of the corresponding signals of the different transmitters.

When the used bandwidth $B$ is sufficient $B$ can also be split into $N$ parts and each transmitter uses only a fraction $B / N$. The drawback of this solution is that the resolution of the system will be reduced and the hardware effort will be higher too, since the filtering of the signals at the receivers has to be done. In addition a separate down-conversion for each band has to be realised at the receiver.

Also a modulation of the phase of each transmitted signal is possible. In this case the separation can be done by correlation of the received signals with the signal of a specific transmitter in order to separate the corresponding pair.

\subsection{Description of arrays in $k$-space}

It can be shown, that a virtual array that is formed by a transmit and a receive array has the same resolution properties as the monostatic array with Tx and Rx placed in the centre of a pair of Tx and Rx antenna of the respective arrays. In the following an example with one transmit antenna and $n$ receive antennas, shown in Fig. 1, is considered. The considerations can be applied to configurations with arbitrary amount of antennas in both arrays. A point scatterer placed at the perpendicular bisector of the Rx array at a distance $r$ is considered.
For a fully monostatic array with $\mathrm{Tx}$ and $\mathrm{Rx}$ antennas at the same position the width of the occupied $k$-space becomes

$$
\begin{aligned}
\Delta k_{y} & =\frac{D}{2 r} \cdot\left|\boldsymbol{k}_{\mathrm{Rx}}+\boldsymbol{k}_{\mathrm{Tx}}\right| \\
& =\frac{D}{r}|\boldsymbol{k}| .
\end{aligned}
$$

The azimuth or $y$-resolution can then be determined approximately by

$$
\begin{aligned}
\Delta y & =\frac{2 \pi}{\Delta k_{\mathrm{tot}}}=\frac{2 \pi}{2 \Delta k_{y}} \\
& =\frac{2 \pi r}{2 D|k|}=\frac{\lambda}{2 D} \cdot r .
\end{aligned}
$$

For the situation in case of the virtual array set up by a single transmit element and an object placed on the perpendicular bisector only the positions of the transmit array elements lead to an occupation of the $k$-space in $y$-direction. For the different elements the projection of the resulting $k$ vector $2 \cdot \boldsymbol{k}_{\mathrm{v}}=\boldsymbol{k}_{\mathrm{T}}+\boldsymbol{k}_{\mathrm{R}}$, shown in Fig. 1, on the $k_{y}$ axis is

$k_{y 1}=\sin \varphi_{1} \cdot|\boldsymbol{k}|=\frac{D}{2 r} \cdot|\boldsymbol{k}|$
$k_{y n}=\sin \varphi_{n} \cdot|\boldsymbol{k}|=\frac{D}{2 r} \cdot|\boldsymbol{k}|$.

Taking the two outer elements into account since they result in the largest extension in $k_{y}$-direction, the size of the occupied $k$-space is

$\Delta k_{y}=k_{y 1}+k_{y N}=\frac{D}{r} \cdot|\boldsymbol{k}|$.

and the resulting azimuth resolution results in

$\Delta y=r \cdot \frac{\lambda}{D}$.

Comparing this with the monostatic case, from in (8)

$\Delta y=r \cdot \frac{\lambda}{D}=r \cdot \frac{\lambda}{2 D_{\mathrm{v}}}$

it turns out that the resolution of the virtual array is the same as for a monostatic array with half the array size: $D=2 \cdot D_{v}$.

\subsection{Realisation of virtual arrays}

For the realisation of a virtual array different possibilities can be considered. In general an equal spacing of the virtual elements is desired. Possible goals could be minimum total space of the overall setup or a specific distribution of the virtual elements with respect to the positions of the real array elements. Setups for those two mentioned realisations are shown Fig. 2. For all realisation a virtual array with $M \cdot N$ virtual elements is generated by $M+N$ antennas. The geometric dimensions can be different for the same virtual extension. 
(a)
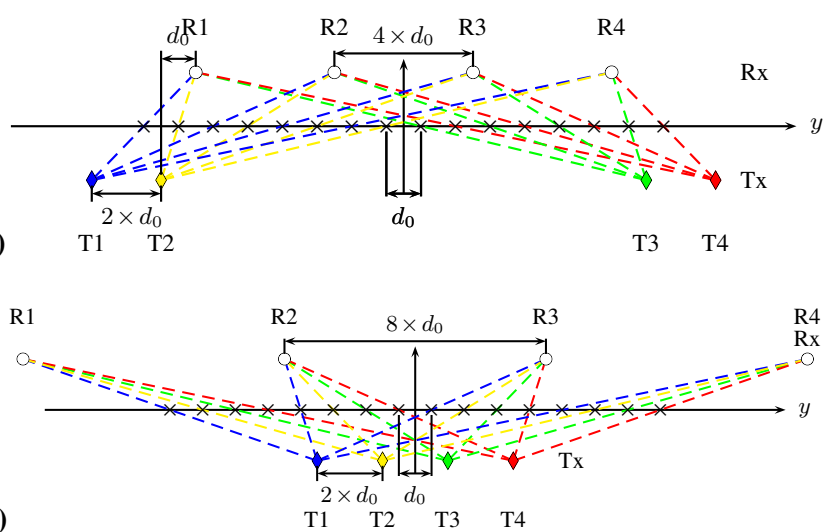

(b)

$\begin{array}{llll}\mathrm{T} 1 & \mathrm{~T} 2 & \mathrm{~T} 3 & \mathrm{~T} 4\end{array}$

Fig. 2. Different options for setting up a virtual array. (a) Placement of Rx- und Tx-antennas according to Wei (2009), Setup 1. (b) Alternative placement of Rx- und Tx-antennas to separate left and right part of the virtual array with a maximum number of virtual elements, Setup 2.

\section{Methods for grating lobe suppression}

\subsection{Choice of the antenna pattern}

A first approach to reduce the influence of objects that are outside the unambiguity interval on this interval, is to adapt the antenna pattern such that the main lobe of the single antenna elements mainly covers the unambiguity interval. Objects outside will then be received already with lower amplitudes. This approach will not be discussed in detail here.

\subsection{Azimuth ambiguity and resolution}

As shown in the previous section the azimuth resolution capabilities depend on the maximum dimensions of the array, see Eq. (13). The more space the elements occupy, the higher the resolution. On the other hand the number of virtual array elements determines the number of azimuth sample points and therefore together with the resolution the interval where scatterers can be localised uniquely. Let $d_{0}$ be the distance between the virtual array elements. The unambiguity interval then becomes

$\varphi_{\text {unamb }}= \pm \sin ^{-1}\left(\frac{c_{0}}{4 f_{\mathrm{c}} d_{0}}\right)$.

When the distance between the virtual elements is e.g. $d_{0}=0.8 \cdot \lambda$, then the unambiguity interval becomes $\varphi_{\text {unamb }}=$ $\pm 18.2^{\circ}$. At an angular offset of

$\Delta \varphi_{\text {grating }}=2 \cdot \arcsin \left(\frac{c_{0}}{4 f_{c 0} d_{0}}\right)$

grating lobes will appear.

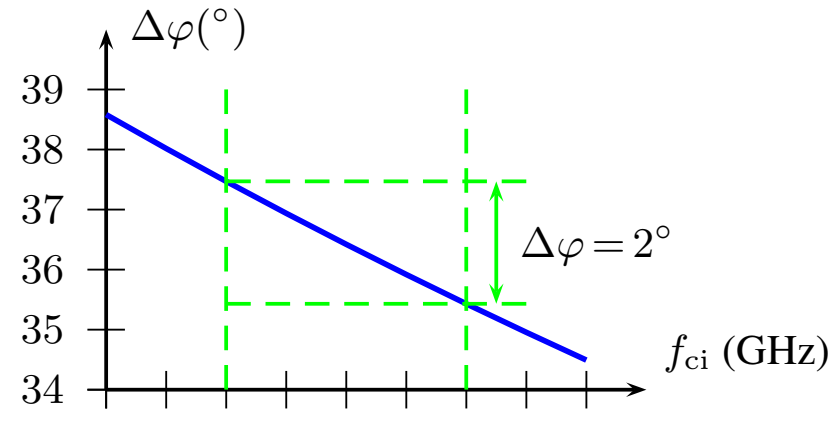

707172737475767778

Fig. 3. Distance between object's position and grating lobe as a function of centre frequency $f_{c i}$ (object at the perpendicular bisector of the array).

\subsection{Evaluation of sub bands}

As shown in Eq. (15) the position of the grating lobes is varying with a change of the centre frequency of the used frequency band. In contrast to this the results of different sub bands overlap at the correct angular position of the object. When the spacing between the virtual elements is expressed in terms of the wavelength $\lambda_{c, 0}$ that corresponds with $f_{c, 0}$ and $d_{0}=\alpha \lambda_{c, 0}$, then the distance between main and grating lobe in another frequency band with a centre frequency of $f_{c, i}$ becomes

$\Delta \varphi_{\text {grating, } \mathrm{i}}=2 \cdot \arcsin \left(\frac{f_{c 0}}{4 f_{c i} \alpha}\right)$.

When the azimuth resolution cell of the array is smaller than the separation of the grating lobes, they will be placed in different resolution cells. The grating lobes can then be distinguished from the main lobe by comparing the responses obtained for two different frequency bands.

In Fig. 3 the distance between grating lobes and main lobe is shown as a function of the centre frequency at the frequency range of interest $f=72 \ldots 78 \mathrm{GHz}$. When e.g. a distance between the virtual elements $d_{0}=0.8 \lambda$ is used, the resolution capabilities in azimuth are around $\Delta \varphi=2^{\circ}$. The distance of the grating lobes for $d_{0}=0.8 \lambda$ would be $\Delta \varphi=2^{\circ}$ when the centre frequencies are separated by $4 \mathrm{GHz}$, such that the grating lobes could be resolved at different positions, when the two sub-bands are evaluated.

\section{Measurements}

\subsection{Evaluation of one band}

For first measurements an existing MMW-sensor operating at a slightly higher frequency range $f=90.5 \ldots 100.5 \mathrm{GHz}$ with $1 \mathrm{Tx}$ and Rx channel is used. In order to simulate the data of an array, the sensor is mounted on a linear positioner and the 


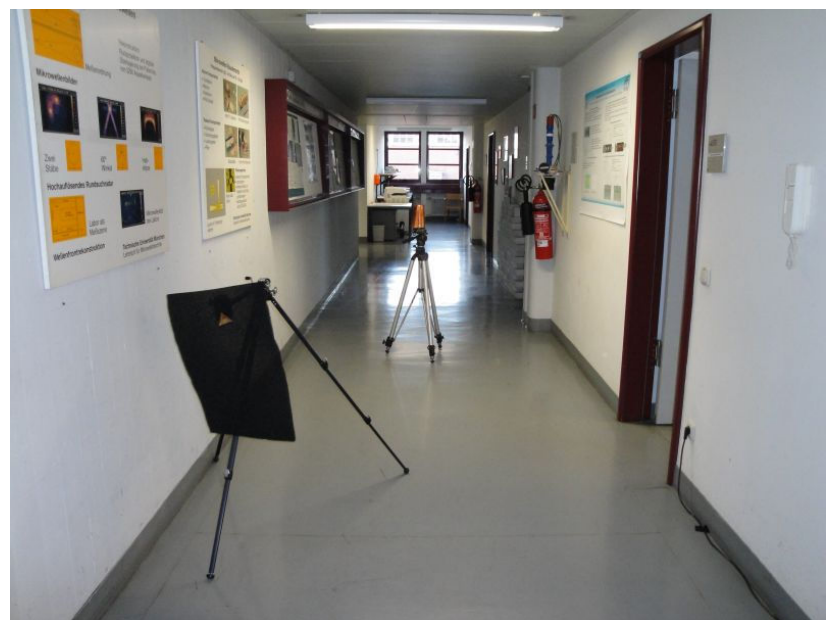

Fig. 4. Measurement scene in a corridor with several objects.

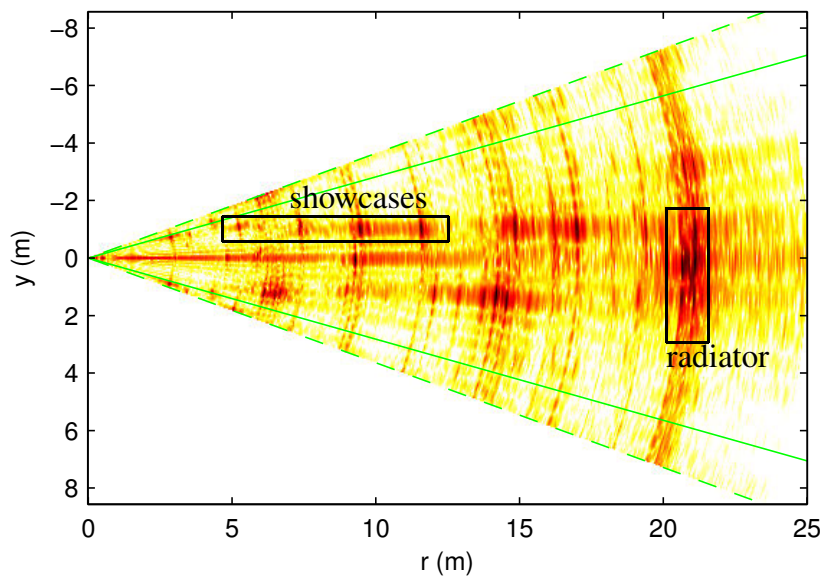

Fig. 5. Reconstruction result of the scene according to the photo shown in Fig. 4, logarithmic scale with $50 \mathrm{~dB}$ dynamic range, green line: unambiguous interval, $f=90.5 \ldots 93.5 \mathrm{GHz}$.

measurement samples are taken at different positions. The measurements are taken in steps of $\Delta y=3 \mathrm{~mm}$, which corresponds to a spacing of $d_{0}=0.92 \times \lambda_{c}$ of the equivalent array. The measurements were done along a corridor of a length of approx. $20 \mathrm{~m}$. A picture of the measured scene is shown in Fig. 4. The major objects that appear are: a trihedral, a copper roll on a mount, a fire extinguisher, showcases and a radiator. The reconstruction result is shown in Fig. 5. The results are represented in a logarithmic scale with $50 \mathrm{~dB}$ dynamic range. The used frequency range for the measurement is $f=90.5 \ldots 93.5 \mathrm{GHz}$.

\subsection{Processing of sub bands}

For the evaluation of sub bands two parts of the sensor's available bandwidth are used. The lower band is $f_{1}=90.5 \ldots 92.8 \mathrm{GHz}$ and the upper one covers $f_{2}=$

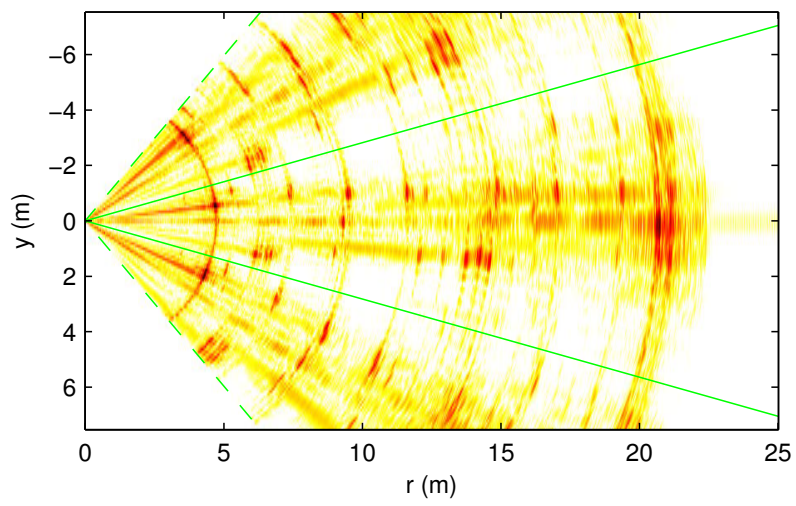

(a)

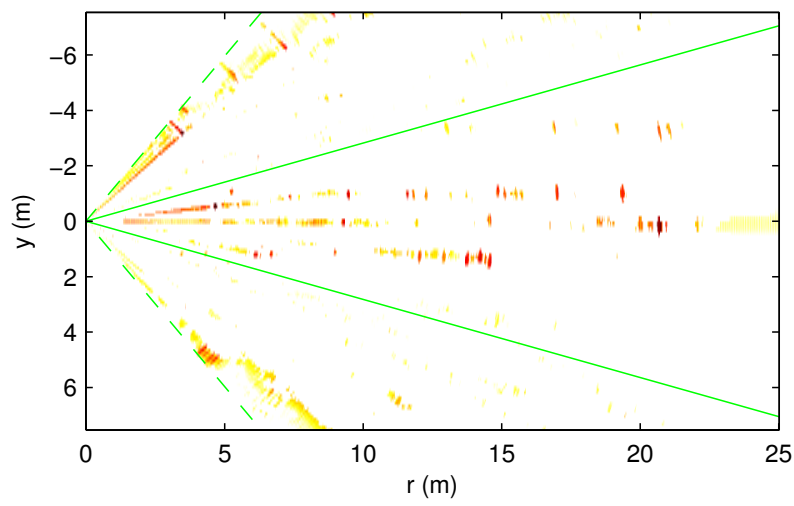

Fig. 6. Comparison of original processed array data and results after applying a mask with the position of the main lobes. (a) Unprocessed measurement result. (b) Result after evaluation of the sub bands.

98.2..100.5GHz. When the two sub bands are evaluated it shows that the result of them don't overlap exactly in range. In addition the grating lobes are not at the exact position in azimuth according to the used frequencies. A possible reason could be inaccuracies in the data acquisition (trigger), but also a mismatch in tuning voltage of the $\mathrm{VCO}$ and expected frequency, which leads to inaccurate output frequencies. As a consequence the evaluation of the measured data has to be adapted. The two images can not be compared point by point at the exact position of a peak. Instead of this a mask is generated, where main-lobes are identified. This is done by determining the object responses produced by grating lobes and main lobes in the two sub-bands. The main lobe is at the same position in the two pictures, grating lobes are at different positions. The mask contains only these points where main lobes have been identified, for other coordinates it is set to zero. A result of this processing scheme is shown in Fig. 6. It shows that outside the unambiguous region grating lobes have been removed for the most part. 


\section{Conclusions}

The concept of virtual arrays has been presented. It is useful to implement arrays with several virtual elements while keeping the hardware effort at a lower level compared to the realisation of an array with $\mathrm{Tx}$ and $\mathrm{Rx}$ at each position of the array. To demonstrate the expected imaging properties an existing MMW-sensor has been used to setup an array with 16 elements. In order to reduce the presence of azimuth ambiguities, two different sub bands have been evaluated. A suppression of grating lobes is possible with this processing step. For the considered bandwidth of $72 \ldots 78 \mathrm{GHz}$ in the realisation of the system, two sub-bands with $2 \mathrm{GHz}$ bandwidth and a separation of the centre frequencies $f_{c i}$ of $4 \mathrm{GHz}$ is considered. Evaluation of sub-bands allows to distinguish between main and grating lobes. Using this approach arrays can be tailored to the desired field of view and a good azimuth resolution.

\section{References}

Wei, M. Digital Antennas, in: Multistatic Surveillance and Reconnaissance: Sensor, Signals and Data Fusion, pages 5-1-5-29, RTO-EN-SET-133, 2009. Available from http://www.rto.nato. int.abstract.aps

Li, J., Stoica, P. MIMO radar signal processing, John Wiley \& Sons Inc., Hoboken, New Kersey, 2009.

Ender, Joachim H. G. MIMO-SAR, in: Proceedings of IRS-07, International Radar Symposium 2007, Kln, Germany, September 2005-2007. 